\title{
O uso do laser de argônio no tratamento da triquíase
}

\author{
Argon laser in the treatment of trichiasis
}

\author{
Nilson Lopes da Fonseca Junior ${ }^{1}$ \\ Lúcia Miriam Dumont Lucci ${ }^{2}$ \\ Leonardo Verri Paulino ${ }^{3}$ \\ José Ricardo Carvalho Lima Rehder ${ }^{4}$
}

\section{RESUMO}

Objetivo: Avaliar a eficácia da técnica de termoablação dos folículos pilosos dos cílios em triquíase com laser de argônio e observar a preferência dos pacientes submetidos a este tratamento, pela anestesia tópica com colírio anestésico ou pela anestesia local injetável. Métodos: Estudo prospectivo de 55 pálpebras de 39 pacientes com triquíase, tratados com fotocoagulação dos folículos pilosos com "Argon green laser" (Alcon ${ }^{\circledR}$ EUA). Neste estudo avaliou-se a idade e o sexo dos pacientes, o número de sessões realizadas, a evolução após as aplicações e a preferência pelo tipo de anestesia. Os parâmetros utilizados foram: (1) Mira - 150 m; (2) Potência-750 mW; (3) Tempo de exposição-0,2 s. Cada cílio recebeu, no máximo, 9 disparos por sessão. Resultados: Dentre os 39 participantes do estudo, 58,9\% eram do sexo feminino e $41 \%$ do sexo masculino. A idade média foi de 71 anos. Houve cura em 69\% (38 pálpebras), sendo 29\% (16 pálpebras) com apenas uma sessão de laser. Houve preferência estatisticamente significativa pelo procedimento realizado sob anestesialocal injetável. Conclusão: Os resultados sugerem que a anestesia local no tratamento da triquíase com laser de argônio é a preferência da maioria dos pacientes e que a termoablação dos folículos pilosos com laser de argônio é tratamento efetivo para a triquíase.

Descritores: Cílios/patologia; Lasers/uso terapêutico; Argônio/uso terapêutico; Fotocoagulação; Anestesia local; Tracoma/terapia

\section{INTRODUÇ̃̃̃O}

Triquíase é uma afecção adquirida dos cílios, normalmente posicionados na lamela anterior palpebral ${ }^{(1-3)}$, que perdem o direcionamento normal ${ }^{(4)}$ e se dirigem para a superfície ocular ${ }^{(5-6)}$.

A triquíase é uma doença freqüente e apresenta as seguintes causas: 1tracoma, 2- inflamações palpebrais ou conjuntivais crônicas, 3- síndrome de Stevens-Johnson, 4- queimaduras químicas, 5- penfigóide ocular, 6- cirurgias palpebrais prévias, 7- Herpes zoster, 8- trauma ${ }^{(1-4,6-8)}$, ou em uma margem palpebral normal ${ }^{(3)}$.

A triquíase é classificada em maior ou menor, conforme a quantidade de cílios mal direcionados. O tracoma é a principal causa de triquíase maior (5 ou mais cílios acometidos $)^{(2,5-6)}$. Um outro método de se avaliar a magnitude da triquíase é quantificando-se o número de segmentos palpebrais envolvidos (1, 2 ou difuso) $)^{(4)}$.

Devido o traumatismo constante dos cílios contra a conjuntiva e a córnea, esta patologia pode causar dor ocular, sensação de corpo estranho constante, blefaroespasmo reflexo, lacrimejamento e congestão conjuntival $^{(1-3,5-6,9-11)}$. Dentre as seqüelas podemos destacar a conjuntivite e a ceratite infecciosa, as erosões corneanas, os abscessos corneanos, vascula- 
rização corneana, opacidades corneanas e a baixa acuidade visual $^{(1-2,4,7,10,12-13)}$.

Existem várias modalidades de tratamento para triquíase: 1- epilação mecânica, 2- eletrólise, 3- crioterapia, 4- cirurgias, 5irradiação, 6- vaporização a laser do folículo piloso ${ }^{(9-10,13-14)}$.

Na epilação mecânica o cílio é removido com uma pinça e o resultado é apenas temporário, observando-se uma recidiva em 2 ou 3 semanas ${ }^{(2-3,6)}$.

A eletrólise e a crioterapia podem apresentar complicações, apesar dos bons resultados ${ }^{(14-17)}$.

A intervenção cirúrgica deve ser reservada para os casos com comprometimento de uma área de margem palpebral extensa ${ }^{(3,5)}$ ou pela grande quantidade de cílios em triquíase nos casos localizados.

Apesar das várias modalidades de tratamento, a recidiva da triquíase é um problema comum ${ }^{(2-3)}$.

A técnica de termoablação dos folículos pilosos das margens palpebrais com laser de argônio tem mostrado excelentes resultados $^{(8-11) .}$ Essa técnica foi descrita por Benny em 1979, proporcionando uma ablação folicular seletiva, segura e realizada com anestesia local ${ }^{(1,3)}$.

Este estudo tem dois objetivos: avaliar a eficácia da técnica de termoablação dos folículos pilosos dos cílios em triquíase com laser de argônio e observar a preferência dos pacientes submetidos a este tratamento, pela anestesia tópica com colírio anestésico ou pela anestesia local injetável com Xilocaína 2\% associada a Epinefrina (1:100.000).

\section{MÉTODOS}

Foram estudadas, prospectivamente, 55 pálpebras (39 pacientes) de portadores de triquíase menor, atendidos no ambulatório de Plástica Ocular da Disciplina de Oftalmologia da Faculdade de Medicina do ABC, tratados com termoablação dos folículos pilosos com "Argon green laser" (Alcon ${ }^{\circledR}$ - EUA). Estes pacientes não foram submetidos a outros tratamentos para a afecção em estudo. Todos os pacientes autorizaram por escrito o procedimento através do termo de esclarecimento e livre consentimento.

Após ter sido diagnosticada a triquíase, o paciente foi avaliado pelos autores conforme os critérios de inclusão (ausência de deformidades e/ou lesões palpebrais). Realizou-se termoablação dos folículos pilosos palpebrais sob anestesia tópica (Proximetacaína colírio) nos casos unilaterais. Nos casos bilaterais, o procedimento foi realizado sob anestesia tópica no olho direito e sob anestesia local injetável (Xilocaína 2\% associada a Epinefrina - 1:100.000) no olho esquerdo, próximo à borda palpebral afetada. Após a realização da anestesia, o paciente foi posicionado na lâmpada de fenda para a aplicação do laser de argônio.

A pálpebra afetada foi retificada a fim de que os cílios pudessem permanecer completamente expostos e o paciente orientado a manter posição do olhar oposta ao foco luminoso. $\mathrm{O}$ laser de argônio foi disparado em posição co-axial ao folículo e sob os seguintes parâmetros: (1) Mira - 150 m; (2) Potência 750 mW; (3) Tempo de exposição - 0,2 s. Foram realizados 2 disparos para causar uma depressão na margem palpebral sobre o cílio a ser tratado e então 9 disparos em cada sessão.

Neste estudo os pacientes que apresentavam blefarite e/ou meibomite foram submetidos a uma higiene palpebral antes da aplicação do laser. Esta higiene foi realizada com anti-sepsia local pela mesma equipe médica que realizou a laserterapia. Nestes casos foram necessários 3 a 4 disparos extras de laser para a formação de uma depressão bem definida.

Após a primeira sessão, foi prescrita pomada oftalmológica de Cloranfenicol + Retinol, 3 vezes ao dia, por 5 dias, e agendado um retorno em 5 semanas. Nos casos bilaterais, foi questionado ao paciente em qual dos dois olhos a dor foi maior, fazendo a seguinte pergunta: “Comparando a dor da anestesia injetável mais a dor da aplicação do laser no olho direito com a dor do colírio anestésico mais a dor da aplicação do olho esquerdo, em qual dos dois olhos a dor for maior?”.

No retorno, após 5 semanas, o paciente foi avaliado e, se necessária, nova sessão de laser de argônio era executada sob a anestesia que proporcionou maior conforto para o paciente, sendo a prescrição e a conduta iguais às realizadas após a primeira sessão.

Quando necessário, uma terceira e última sessão foi realizada, mantendo-se a mesma prescrição e intervalo para retorno.

Nos casos em que não houve sucesso, transcorridas as 5 semanas após a terceira sessão, foi indicada intervenção cirúrgica para a retirada dos cílios em triquíase e enxerto de mucosa labial.

Os pacientes tratados sem necessidade de intervenção cirúrgica receberam alta ambulatorial após período de seguimento de aproximadamente 3 meses.

Durante este estudo avaliou-se a idade e o sexo dos pacientes, número de sessões realizadas, a evolução após as aplicações e a preferência dos pacientes com triquíase bilateral submetidos a este tratamento, pela anestesia tópica com colírio anestésico ou pela anestesia local injetável.

\section{MÉTODOS}

Utilizou-se o teste do $\mathrm{X}^{2}$ (qui-quadrado) para amostras não independentes, pressupondo-se que as freqüências esperadas são distribuídas de modo eqüitativo. O nível de rejeição para a hipótese de nulidade foi fixado sempre em um valor igual ou menor que $0,005(5 \%)^{(18)}$. Quando a estatística calculada apresentou significância utilizou-se um asterisco (*) para caracterizá-la e em caso não significante utilizou-se NS.

\section{RESULTADOS}

Dentre os 39 participantes deste estudo, 23 pacientes $(58,9 \%)$ eram do sexo feminino e 16 (41\%) do sexo masculino. A idade dos pacientes variou de 45 a 88 anos (média de 71 
anos). Com relação à lateralidade, 23 pacientes apresentavam triquíase unilateral.

Observou-se um percentual de sucesso no tratamento da triquíase com laser de argônio em 38 pálpebras (69\%) e houve indicação cirúrgica em 17 pálpebras (30,9\%) (Gráfico 1).

Em 16 pálpebras $(42,1 \%)$ a cura foi alcançada após uma única sessão; em 14 pálpebras (36,8\%) foram necessárias duas sessões; e, em 8 pálpebras (21\%), três sessões (Gráfico 2).

Houve 16 casos bilaterais, dos quais 13 pacientes $(81,2 \%)$ referiram menor dor ao procedimento com anestesia local e 3 pacientes (18,7\%) com anestesia tópica (Gráfico 3).

Quanto à causa da triquíase, o tracoma cicatricial foi o principal agente $(64,1 \%)$.

Não se observou complicações anatômicas nas pálpebras ou alterações na pigmentação da pele devido ao uso do laser.

\section{DISCUSSÃO}

A causa mais comum de triquíase é o tracoma, sendo a segunda causa de cegueira mundial segundo a Organização Mundial de Saúde (OMS). O traumatismo crônico e constante dos cílios na superfície ocular, leva a alterações corneanas que causam baixa acuidade visual ${ }^{(19)}$.

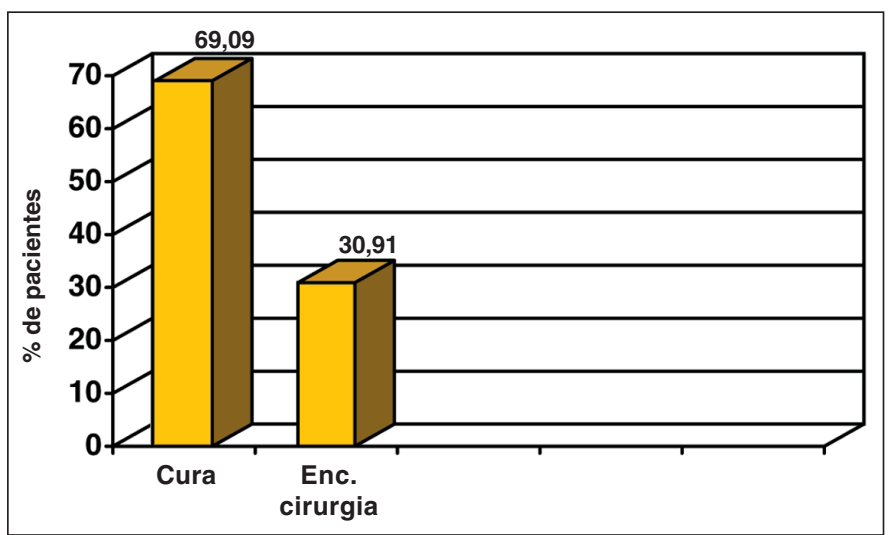

Gráfico 1 - Evolução dos pacientes submetidos a termoablação com laser de argônio no tratamento da triquíase, Faculdade de Medicina do $A B C, 2001$



Gráfico 2 - Distribuição do número de sessões necessárias para cura dos pacientes com triquíase tratados com termoablação, Faculdade de Medicina do ABC, 2001

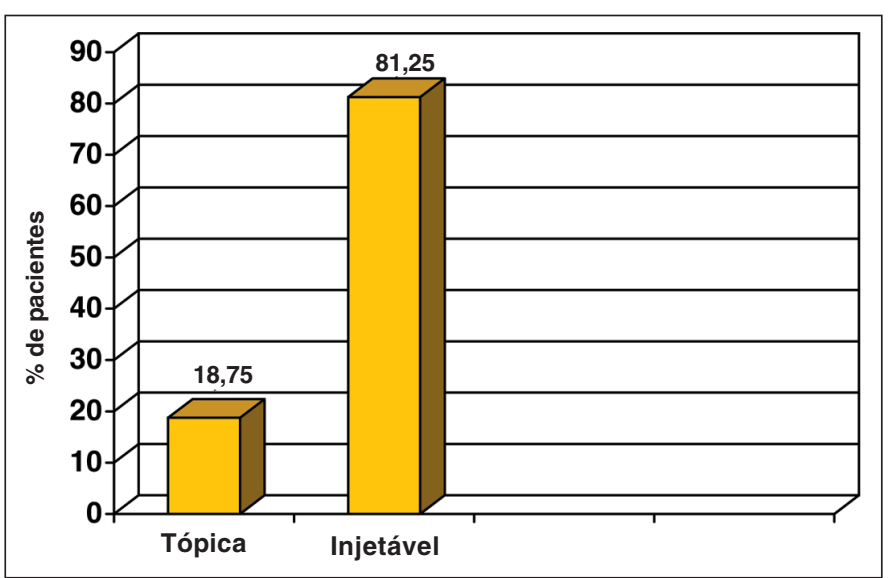

Gráfico 3 - Prefência pela anestesia tópica ou injetável nos 16 casos bilaterais de triquíase, tratados com termoablação no Ambulatório de Plástica Ocular da Faculdade de Medicina do ABC, 2001

Existem vários estudos sobre correção da triquíase com diferentes técnicas e resultados. O objetivo é retirar o cílio inteiro para prevenir sua recidiva. Dentre as técnicas descritas, a crioterapia está contra-indicada nos casos de penfigóide, pela possibilidade de recidiva da doença. Hipopigmentação cutânea palpebral é uma complicação comum com o uso desta técnica, além da destruição de cílios normais, necrose palpebral, úlcera corneana, xerose, celulite, reativação de herpes. A crioterapia atinge índices de complicações de até $26 \%{ }^{(17)}$. A cirurgia requer local adequado para o procedimento, que deve ser realizado em centro cirúrgico com utilização de microscópio.

Em relação à eletrólise, as recidivas são freqüentes podendo atingir até $60 \%$ dos casos, sendo indicada apenas em casos com pequeno número de cílios $^{(15)}$. Dentre as complicações desta técnica podem ser citadas as cicatrizes e distorções palpebrais ${ }^{(15)}$.

A técnica de termoablação, para o tratamento da triquíase, baseia-se na destruição do folículo piloso anormal pelos efeitos da fotocoagulação e fotovaporização dos raios laser ${ }^{(20)}$. A fotocoagulação com laser de argônio, resulta da transformação da energia luminosa em energia térmica, através da absorção da luz pelo pigmento, com a modificação das proteínas teciduais ${ }^{(1,3)}$.

Foram utilizados os seguintes parâmetros para os disparos baseado em experiências anteriores: (1) Mira - 150 mm; (2) Potência - 750 mW; (3) Tempo de exposição - 0,2 s. Foram aplicados, no máximo, 9 disparos por cílio em cada sessão, apesar de alguns trabalhos preconizarem mais de 35 disparos por cílio em cada sessão ${ }^{(1,3)}$, e apresentando índice de cura de $37,7 \%$ após uma sessão ${ }^{(1)}$. Oshry et al preconizaram 15 a 20 disparos por cílio em cada sessão, tendo um índice de $80 \%$ de cura $^{(2)}$. Nesses estudos os parâmetros utilizados foram semelhantes: (1) Mira - 50 m; (2) Potência - 800-1400 mW; (3) Tempo de exposição - 0,2 s.

Os resultados deste estudo podem ser explicados pela diferença dos parâmetros utilizados (menor potência e maior 
mira) e pelo número de disparos realizados por sessão. Neste trabalho 69\% dos pacientes submetidos a termoablação com laser de argônio para o tratamento da triquíase foram curados $\left(\mathrm{X}_{\text {calc }}^{2}=8,018 *\right)$, sendo que $42,1 \%$ foram curados com uma sessão; $36,8 \%$ com duas sessões e $21 \%$ após três sessões $\left(\mathrm{X}_{\text {calc }}^{2}=2,729 \mathrm{NS}\right)$.

Neste estudo preconizou-se um profundidade de $2 \mathrm{~mm}$ com os disparos do laser para uma efetiva vaporização do folículo piloso em pálpebra superior e inferior. Após análise dos resultados obtidos em um estudo piloto no nosso serviço, constatou-se que 9 disparos do laser por cílio são suficientes para atingir uma profundidade aproximada de $2 \mathrm{~mm}$. Um menor número de disparos, comparados com outros estudos, ocasiona uma menor lesão tecidual local e uma menor chance de causar defeitos na margem palpebral.

Outros tratamentos existentes para a triquíase mostram índices de cura de: 56 a $90 \%$ na crioterapia ${ }^{(16)}$ e 50 a $80 \%$ na eletrólise $^{(15)}$.

Quanto ao procedimento anestésico para a termoablação com laser de argônio no tratamento da triquíase, a maioria dos estudos preferem a anestesia injetável ${ }^{(3,6,11,21)}$. Existem relatos de aplicação de laser sem anestesia ${ }^{(8)}$ e sob anestesia tópica ${ }^{(1)}$. Neste estudo observou-se uma preferência pela anestesia injetável com Xilocaína 2\% associada a Epinefrina (1:100.000) em cerca de $81 \%$ dos pacientes com triquíase bilateral $\left(\mathrm{X}_{\text {calc }}=\right.$ $6,250 *)$. Nestes pacientes, na primeira sessão, a pálpebra direita foi submetida ao procedimento sob anestesia tópica (Proximetacaína colírio) e a pálpebra esquerda sob anestesia injetável (Xilocaína 2\% associada a Epinefrina - 1:100.000). No término da primeira sessão perguntou-se ao paciente qual tipo de anestesia foi preferida. Quando necessário, as demais sessões foram realizadas sob a anestesia preferida pelo paciente.

Alguns trabalhos relatam complicações na aplicação de laser de argônio como defeito da margem palpebral e a hipopigmentação ${ }^{(20-21)}$, porém neste estudo não foram observadas tais complicações.

Apesar de algumas desvantagens da laserterapia no tratamento da triquíase como a difícil execução em pacientes nãocooperativos e/ou com tremores ${ }^{(3)} \mathrm{e}$ a impossibilidade de execução em pacientes acamados, as vantagens mostram-se superiores $^{(22)}$. Uma outra desvantagem desta técnica é o alto custo do aparelho.

Dentre as vantagens deste tipo de tratamento podemos destacar a seletividade do procedimento ${ }^{(3)}$, dano tecidual mínimo dificilmente causando complicações ${ }^{(8)}$, alto índice de sucesso e baixa incidência de recorrência ${ }^{(1-3,8,22)}$.

\section{CONCLUSÃO}

Os resultados mostram que a termoablação dos folículos pilosos com laser de argônio utilizando-se 9 disparos é um método eficaz para o tratamento da triquíase. Os pacientes do presente estudo preferiram o procedimento sob anestesia local injetável.

\begin{abstract}
Purpose: To evaluate the effectiveness of thermoablation of eyelashes in trichiasis with Argon laser and to observe the patients' choice of topic or local anesthesia. Methods: Prospective study of 55 eyelids of 39 patients with trichiasis treated with photocoagulation of the eyelash follicle with Argon green laser (Alcon ${ }^{\circledR}$ - USA). Age, sex, number of applications in each session, evolution after the application and preference for the anesthesic method were evaluated. The laser settings were: $150 \mu \mathrm{m}$ aim $750 \mathrm{~mW}$ potency; 0.2 seconds exhibition. Each eyelash received no more than 9 applications per procedure. Results: Of the 39 patients, $58.9 \%$ were female and $41 \%$ were male. Mean age was 71 years. There was resolution in 69\% (38 patients), and 29\% (16 eyelids) underwent a single procedure. The preference for local anesthesia is statistically significant. Conclusion: The results suggest that most patients chose local anesthesia for the treatment of trichiasis with argon laser and the thermoablation of eyelashes with argon green laser is an effective treatment of trichiasis.
\end{abstract}

Keywords: Cilia/pathology; Lasers/therapeutic use; Argon/ therapeutic use; Light coagulation; Anesthesia, local; Trachoma/therapy

\section{REFERÊNCIAS}

1. Hata MM, Monteiro ECL, Schellini SA, Aragon FF, Padovani CR. Laser de argônio no tratamento da triquíase e da distiquíase. Arq Bras Oftalmol 1999; 62:285-95.

2. Oshry T, Rosenthal G, Lifshitz T, Shani L, Yassur Y. Argon green laser photoepilation in the treatment of trachomatous trichiasis. Ophthal Plast Reconstr Surg 1994;10:253-5.

3. Dantas RRA. Triquíase - fotocoagulação com laser argônio. Rev Bras Oftalmol 1992;51:9-12.

4. Araújo FAM, Cruz AAV. Alterações de cílios no Hospital das Clínicas da Faculdade de Medicina de Ribeirão Preto-USP. Arq Bras Oftalmol 2002;65: 343-9.

5. Scheie HG, Albert DM. Distiachiasis and trichiasis: origin and management. Am J Ophthalmol 1966;61:718-20.

6. Gossman MD, Yung R, Berlin AJ, Brightwell JR. Prospective evaluation of the argon laser in the treatment of trichiasis. Ophthalmic Surg 1992;23:183-7.

7. Sahni J, Clark D. Argon laser and trichiasis: a helpful tip. Br J Ophthalmol 2001;85:762.

8. Sharif KW, Arafat AF, Wykes WC. The treatment of recurrent trichiasis with argon laser photocoagulation. Eye 1991;5:591-5.

9. Berry J. Recurrent trichiasis: treatment with laser photocoagulation. Ophthalmic Surg 1979;10:36-8.

10. Awan KJ. Argon laser treatment of trichiasis. Ophthalmic Surg 1986;17:658-60.

11. Bartley GB, Lowry JC. Argon laser treatment of trichiasis. Am J Ophthalmol 1992;113:71-4.

12. Reacher MH, Munoz B, Alghassany A, Daar AS, Elbualy M, Taylor HR. A controlled trial of surgery for trachomatous trichiasis of the upper lid. Arch Ophthalmol 1992;110:667-74.

13. Hartzler J, Neldner KH, Forstot SL. X-ray epilation for the treatment of trichiasis. Arch Dermatol 1984;120:620-4.

14. Sullivan JH, Beard C, Bullock JD. Cryosurgery for treatment of trichiasis. Am J Ophthalmol 1976;82:117-21.

15. Creppe MC, Silva MRBM, Schellini SA. Tratamento por eletrólise do mal posicionamento dos cílios palpebrais. Rev Bras Oftalmol 1996;55:595-600. 
16. Rice CD, Kersten RC, al-Hazzaa S. Cryotherapy for trichiasis in trachoma. Arch Ophthalmol 1989;107:1180-2.

17. Wood JR, Anderson RL. Complications of cryosurgery. Arch Ophthalmol 1981;99:460-3.

18. Siegel S. Estatística não paramétrica para as ciências do comportamento. São Paulo: McGraw-Hill do Brasil;1975.

19. Whitcher JP, Srinivasan M, Upadhyay MP. Corneal blindness: a global perspective. Bull World Health Organ 2001;79:214-21.
20. Awan KJ. Laser photocoagulation - vaporization therapy of trichiasis. Ophthalmic Laser Ther 1988;3:3-10.

21. Yung CW, Massicotte SJ, Kuwabara T. Argon laser treatment of trichiasis: a clinical and histopathologic evaluation. Ophthal Plast Reconstr Surg 1994; 10:130-6.

22. Basar E, Ozdemir H, Ozkan S, Cicik E, Mirzatas C. Treatment of trichiasis with argon laser. Eur J Ophthalmol 2000;10:273-5.

\section{Congresso \\ da Sociedade Norte-Nordeste de Oftalmologia}

\section{0 de Junho a 3 de Julho de 2004 Rio Poti Hotel - Teresina - PI}

\section{Informações: Agenda Promoções Ltda. Fone/Fax: (86) 223-9444 \\ E-mail: oftalmologia.nortenordeste.2004@bol.com.br Home-page: www.agendapromocoes.com.br}

